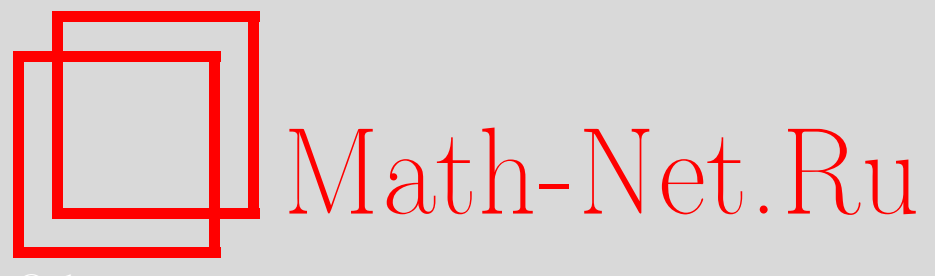

О. А. Задорожнюк, Нижняя оценка сложности реализации одной булевой функции двуслойными контактными схемами на плоской целочисленной решетке, Дискрет. матем., 1997, том 9, выпуск 2, 40-52

DOI: https://doi.org/10.4213/dm477

Использование Общероссийского математического портала Math-Net.Ru подразумевает, что вы прочитали и согласны с пользовательским соглашением http://www.mathnet.ru/rus/agreement

Параметры загрузки:

IP : 54.198 .64 .247

26 апреля 2023 г., 03:42:09 


\title{
Нижняя оценка сложности реализащии одной булевой функщии двуслойными контактными схемами на плоской целочисленной решетке
}

\author{
(c) 1997 г. О. А. Задорожнюк
}

\begin{abstract}
В данной работе изучается сложность $L_{d}\left(f_{n}\right)$ реализации индивидуальных булевых функций плоскими двуслойными контактными схемами [2]. Функциональная часть таких схем содержит контакты, проводники и изоляторы. Кроме того, имеется специальная плоская схема, состоящая лишь из проводников и изоляторов, которая моделирует подачу управляющих воздействий (значений переменных) на контакты функциональной части. В качестве меры сложности рассматривается площадь, занимаемая схемой. В статье для универсальной функции Нечипорука получена оценка вида $L_{d}\left(f_{n}\right) \geqslant C n^{2} / \log _{2} n$, где $C$ - некоторая постоянная.
\end{abstract}

\section{1. Введение}

Развитие в электронике интегральной технологии обусловило интерес к различным моделям плоских функциональных схем. В данной работе рассматриваются модели плоских контактных схем, для которых мерой сложности может служить площадь, занимаемая схемой. Одна из таких моделей, прямоугольная контактная схема, уложенная на целочисленную решетку, рассматривалась Ю. Г. Таразевичем в [1]. В дальнейшем схемы из [1] будем называть однослойными схемами. В [2] была предложена модель двуслойной контактной схемы. В ней в дополнение к функциональному (нижнему) слою, являющемуся по сути однослойной схемой, вводился еще один, верхний слой схемы, который обеспечивал подачу управляющих воздействий (значений переменных) на активные элементы (контакты) нижнего слоя, подобно тому, как это делается в реальных интегральных схемах. Наличие верхнего слоя существенно влияет на структуру, а следовательно, и на сложность схемы. Так функция Шеннона, характеризующая сложность однослойных схем, равна по порядку $2^{n} / \log n$ [3], в то время как для двуслойных схем ее порядок равен $2^{n}$ [2] (здесь и далее логарифм берется по основанию 2). Представляет определенный интерес рассмотреть влияние ограничений, связанных с двуслойными схемами, на сложность реализации явно задаваемых булевых функций. В настоящей статье для функции Э. И. Нечипорука [4] получена нижняя оценка сложности реализации двуслойными контактными схемами, равная по порядку $n^{2} / \log n$. Заметим, что 
в классе однослойных схем для этой функции этим же методом можно получить нижнюю оценку порядка $n^{2} /(\log n \log \log n)$, а в классе обычных контактных схем, где мерой сложности служит число контактов в схеме, ее сложность оценена снизу по порядку как $(n / \log n)^{2}[4]$.

\section{2. Двуслойные контактные схемы на плоской целочисленной решетке}

Пусть $\left(V_{1}, E_{1}\right)$ - плоская целочисленная решетка, $V_{1}$ - множество всех точек евклидовой плоскости с целочисленными координатами, а $E_{1}-$ множество всех отрезков единичной длины с концами в $V_{1}$. Элементы из $V_{1}$ и $E_{1}$ будем называть, соответственно, вершинами и ребрами. Будем далее рассматривать прямоугольные сети, являющиеся конечными подграфами $\left(V_{1}, E_{1}\right)$, назовем их $r$-сетями. Некоторые вершины $r$-сети (не обязательно различные) пометим символами $A, B, C, \ldots$ и назовем их полюсами. Пусть задан конечный алфавит

$$
X=\left\{x_{1}, \ldots, x_{n}, \bar{x}_{1}, \ldots, \bar{x}_{n}, 0,1\right\}
$$

Определение 1. Схемой нижнего слоя будем называть $r$-сеть, каждому ребру которой приписан один из символов алфавита $X$, а некоторые вершины помечены символами $A, B, C, \ldots$

Ребра, помеченные символами $x_{i}\left(\bar{x}_{i}\right)$ будем называть замыкающими (размыкающими) контактами; ребра, помеченные символами 0 (1) - изоляторами (проводниками); помеченные вершины - полюсами.

Будем рассматривать лишь такие схемы нижнего слоя, каждый полюс которых лежит на периметре схемы.

Рассмотрим цепи, соединяющие пары полюсов схемы нижнего слоя. Каждой цепи $Z=\left\{a_{1}, a_{2}, \ldots, a_{s}\right\}$ поставим в соответствие конъюнкцию $K_{Z}=b_{1} \& b_{2} \& \ldots \& b_{s}$, где $b_{i}$ - символ, приписанный ребру $a_{i}, i=1, \ldots, s$.

Каждой паре полюсов, например, $A$ и $B$, схемы нижнего слоя ставится в соответствие булева функция

$$
f_{A B}\left(x_{1}, \ldots, x_{n}\right)=\bigvee_{Z} K_{Z}
$$

где дизъюнкция берется по всем цепям, соединяющим полюса $A$ и $B$. Если полюса $A$ и $B$ совпадают, то полагаем $f_{A B} \equiv 1$.

Заметим, что описанные выше схемы нижнего слоя практически ничем не отличаются от модели Ю. Г. Таразевича.

Рассмотрим теперь плоскую решетку $\left(V_{2}, E_{2}\right)$, полученную из $\left(V_{1}, E_{1}\right)$ следующим сдвигом: каждая вершина $v, v \in V_{1}$, имеющая координаты $(x, y)$, отображается в вершину $v^{\prime}$ с координатами $(x+1 / 2, y+1 / 2)$.

Будем говорить, что ребро $e_{1}$ решетки $\left(V_{1}, E_{1}\right)$ покрывается ребром $e_{2}$ решетки $\left(V_{2}, E_{2}\right)$, если $e_{2}$ пересекает $e_{1}$.

Определение 2. Схемой верхнего слоя будем называть $r$-сеть, принадлежащую решетке $\left(V_{2}, E_{2}\right)$, каждому ребру которой приписан либо 0, либо 1 . 


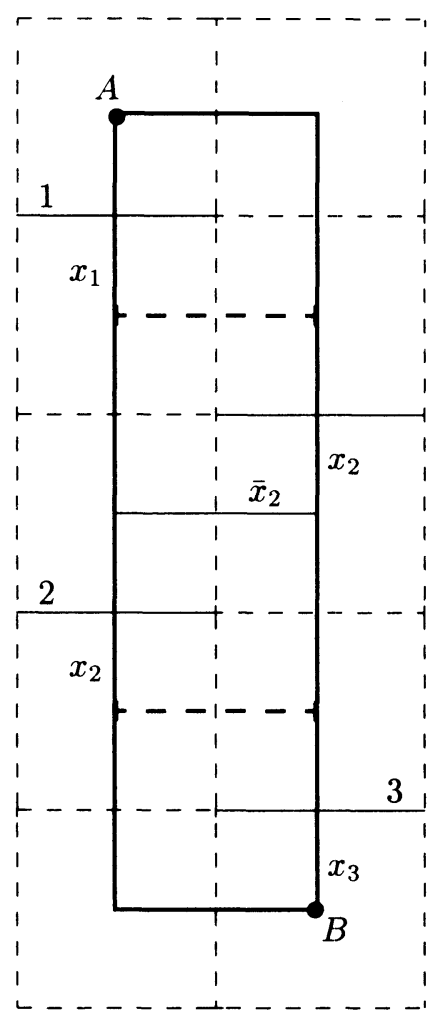

Рис. 1.

Таким образом, в качестве схем верхнего слоя рассматриваются схемы, состоящие только из изоляторов и проводников. В дальнейшем будем рассматривать только такие схемы верхнего слоя, все проводники которых могут быть разбиты ровно на $n$ связных компонент, не пересекающихся ни по ребрам, ни по вершинам, и каждая из компонент содержит, по крайней мере, одну вершину, лежащую на периметре схемы. Занумеруем компоненты числами от 1 до $n$.

Определение 3. Плоской двуслойной контактной схемой будем называть всякую пару $\left(S^{1}, S^{2}\right)$, где $S^{1}$ и $S^{2}$ - схемы, соответственно, нижнего и верхнего слоя, для которой выполнены следующие условия: каждое ребро схемы $S^{1}$ покрывается каким-либо ребром схемы $S^{2}$, причем все контакты $x_{i}$ и $\bar{x}_{i}$ покрываются проводниками $i$-й компоненты схемы $S^{2}$.

На рисунках для наглядности контакты и проводники будем обозначать сплошными линиями, а изоляторы пунктирными; ребра схемы нижнего слоя будем изображать жирными линиями, а верхнего тонкими.

Пусть задана плоская двуслойная схема $S=\left(S^{1}, S^{2}\right)$, и схема верхнего слоя $S^{2}$ имеет длину $l$ и высоту $h$. Сложностью схемы $S$ будем считать величину $l h$ и 
обозначать ее через $L_{d}(S)$. Обычным образом введем функцию

$$
L_{d}(f)=\min L_{d}(S)
$$

где минимум берется по всем схемам $S$, реализующим функцию $f$.

На рис.1 представлена двуслойная схема, реализующая функцию

$$
m\left(x_{1}, x_{2}, x_{3}\right)=x_{1} x_{2} \vee x_{1} x_{3} \vee x_{2} x_{3}
$$

\section{3. Обобщенные двуслойные контактные схемы}

В этом параграфе вводится понятие обобщенной двуслойной схемы, в данном случае более удобной для получения нижних оценок.

Пусть конечный связный планарный граф $G_{1}$ (мы не требуем отсутствия петель и кратных ребер) уложен на плоскости.

Определение 4. Обобщенной схемой нижнего слоя будем называть граф $G_{1}$, каждому ребру которого приписан один из символов алфавита $X$, а некоторые вершины (не обязательно различные) помечены символами $A, B, C, \ldots$ и объявлены полюсами.

Функционирование обобщенной схемы нижнего слоя определяется в точности так же, как и функционирование схемы нижнего слоя на целочисленной решетке. В дальнейшем будем рассматривать только двухполюсные схемы.

Граф $G_{1}$ разделяет плоскость на области $T_{0}, \ldots, T_{q}$, из которых $T_{0}$ - внешняя, а остальные - внутренние. Построим граф $G_{2}$, двойственный к графу $G_{1}$. Внутри каждой области $T_{i}$ построим вершину $v_{i}$. Множество этих вершин образует множество вершин графа $G_{2}$. Для каждого ребра $e_{1}$ графа $G_{1}$ построим ребро $e_{2}$ графа $G_{2}$ следующим образом: если ребро $e_{1}$ разделяет области $T_{i}$ и $T_{j}$, то ребро $e_{2}$ соединяет вершины $v_{i}$ и $v_{j}$ и пересекает ребро $e_{1}$ (заметим, что по обе стороны ребра $e_{1}$ может находиться одна и та же область $T_{i}$, в этом случае ребро $e_{2}$ образует петлю). Будем говорить, что ребро $e_{1}$ покрывается ребром $e_{2}$. Очевидно, граф $G_{2}$ также является планарным.

Степенью вершины $v$ будем называть сумму числа ребер и удвоенного числа петель инцидентных $v$.

Определение 5. Помеченный планарный граф $R$ будем называть разводкой, если он имеет следующий вид: все вершины $R$ суть точки некоторой окружности, помеченные в порядке обхода окружности по часовой стрелке, начиная с любой вершины, а все ребра $R$ - хорды этой окружности.

Пусть граф $G_{2}$ уложен на плоскости и $v$ - его вершина степени $m$. Занумеруем инцидентные $v$ ребра (концы петель) числами от 1 до $m$ в порядке обхода вершины по часовой стрелке, начиная с любого ребра. Сопоставим вершине $v$ некоторую $m$-вершинную разводку $R$, причем ребру (концу петли) с номером $i$ сопоставляется вершина $R$ с тем же номером. Будем говорить, что разводка $R$ покрывает вершину $v$.

Пусть $V\left(G_{2}\right)$ - множество вершин графа $G_{2}$ и каждая вершина $v_{i} \in V\left(G_{2}\right)$ покрыта некоторой разводкой $R_{i}$. 
Определение 6. Множество $T=\left\{R_{i}: 1 \leqslant i \leqslant\left|V\left(G_{2}\right)\right|\right\}$ разводок, покрывающих все вершины графа $G_{2}$, будем называть трассировкой графа $G_{2}$.

Пусть $T$ - трассировка графа $G_{2}, v$ - вершина графа $G_{2}$, покрытая разводкой $R$, и $e_{1}$ и $e_{2}$ - два ребра инцидентных $v$.

Определенге 7. Ребра $e_{1}$ и $e_{2}$ будем называть соседними, если соответствующие им вершины в разводке $R$ соединены ребром.

Определение 8. Упорядоченное множество ребер $\left\{e_{1}, \ldots, e_{s}\right\}$ графа $G_{2}$ будем называть $T$-цепью, если для всех $i$ от 1 до $s-1$ ребра $e_{i}$ и $e_{i+1}$ соседние.

Определение 9. Множество ребер $\left\{e_{1}, \ldots, e_{s}\right\}$ графа $G_{2}$ будем называть $T$-компонентой, если любые два ребра из этого множества принадлежат какой-либо одной $T$-цепи.

Определение 10. Множество ребер $\left\{e_{1}, \ldots, e_{s}\right\}$ графа $G_{2}$ будем называть максимальной $T$-компонентой, если оно является $T$-компонентой и не существует другой $T$-компоненты, содержащей данную.

В дальнейшем будем рассматривать только такие трассировки, которые разбивают множество ребер графа $G_{2}$ ровно на $n$ максимальных $T$-компонент. Занумеруем $T$-компоненты числами от 1 до $n$.

Определение 11. Обобщенной схемой верхнего слоя будем называть граф $G_{2}$ с выбранной для него трассировкой $T$.

Определение 12. Обобщенной цвуслойной контактной схемой будем называть всякую пару $\left(S_{0}^{1}, S_{0}^{2}\right)$, где $S_{0}^{1}$ и $S_{0}^{2}$ - обобщенные схемы соответственно нижнего и верхнего слоя, для которой выполнены следующие условия: каждое ребро схемы $S_{0}^{1}$ покрывается каким-либо ребром схемы $S_{0}^{2}$, причем все контакты $x_{i}$ и $\bar{x}_{i}$ покрываются проводниками $i$-й $T$-компоненты схемы $S_{0}^{2}$.

Определение 13. Пустой схемой будем называть схему, нижний слой которой состоит из двух полюсов, а верхний из одной вершины. Функция, реализуемая пустой схемой, тождественно равна нулю.

Сложностью обобщенной схемы будем считать число ребер в нижнем слое и обозначать ее через $L_{g}(S)$. Будем также рассматривать функцию $L_{g}(f)$, определяемую аналогично функции $L_{d}(f)$.

Пусть $e_{1}$ - ребро графа $G_{1}$. Рассмотрим следующие преобразования схемы нижнего слоя.

(1) Удаление ребра $e_{1}$. Ребро $e_{1}$ удаляется.

(2) Замыкание ребра $e_{1}$. Если ребро $e_{1}$ не образует петли, то инцидентные ему вершины стягиваются в одну. Ребро $e_{1}$ удаляется. 

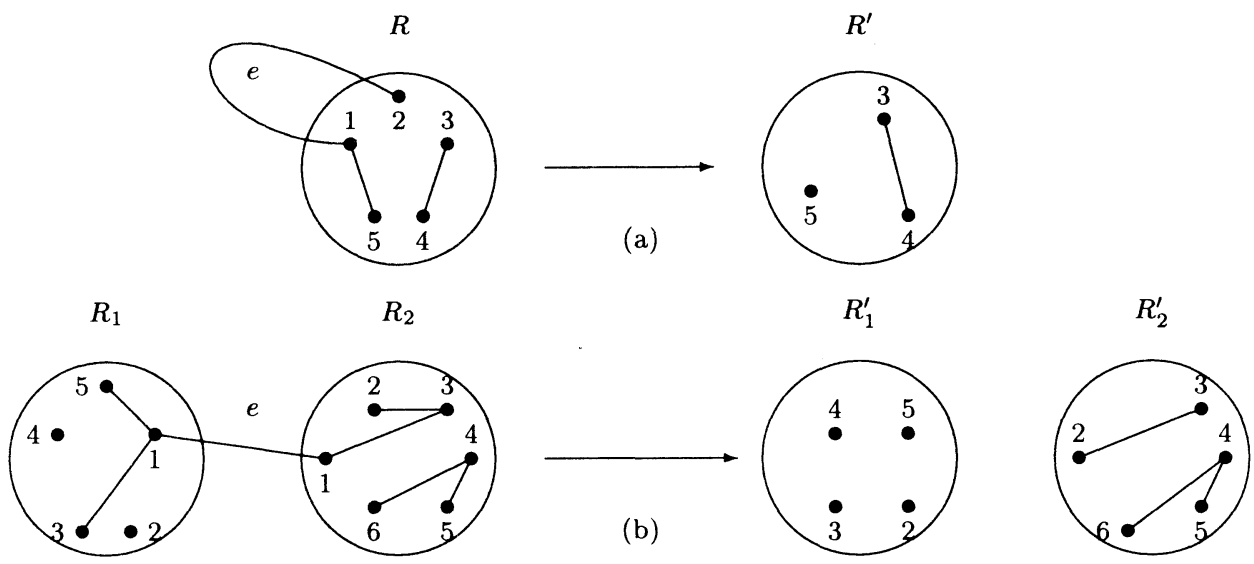

$R$
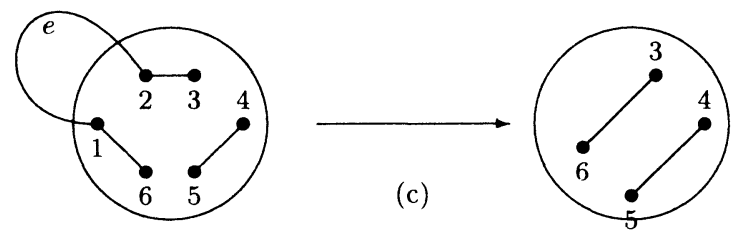

$R_{1}$

$R_{2}$

$R^{\prime}$
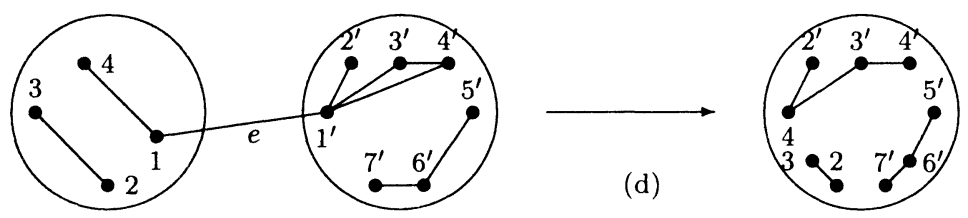

Рис. 2.

Пусть $e_{2}$ - ребро графа $G_{2}$. Его концами являются вершины $v_{1}$ и $v_{2}$. Их покрывают разводки $R_{1}$ и $R_{2}$. Ребру $e_{2}$ в разводках $R_{1}$ и $R_{2}$ соответствуют вершины $v_{1}^{\prime}$ и $v_{2}^{\prime}$. Заметим, что если ребро $e_{2}$ образует петлю, то вершины $v_{1}$ и $v_{2}$ совпадают. В этом случае вершину и покрывающую ее разводку обозначим, соответственно, через $v$ и $R$. Вершины $v_{1}^{\prime}$ и $v_{2}^{\prime}$ всегда различны. Рассмотрим два типа преобразований обобщенной схемы верхнего слоя.

(1) Удаление ребра $e_{2}$. Из разводок $R_{1}$ и $R_{2}$ (из разводки $R$, если ребро $e_{2}$ образует петлю) удаляются вершины $v_{1}^{\prime}$ и $v_{2}^{\prime}$ и инцидентные им ребра (рис. $\left.2 \mathrm{a}, \mathrm{b}\right)$. Ребро $e$ удаляется из графа $G_{2}$. Если при этом появились изолированные вершины, то они удаляются, а из трассировки удаляются покрывающие их разводки.

(2) Замыкание ребра $e_{2}$. 
(a) Ребро $e_{2}$ образует петлю. Ребро $e_{2}$ удаляется из графа $G_{2}$. Из разводки $R$ удаляются вершины $v_{1}^{\prime}$ и $v_{2}^{\prime}$ и инцидентные им ребра. Пусть полученная разводка $R^{\prime}$ состоит из $t$ связных компонент, при этом в $s$ связных компонентах, $1 \leqslant s \leqslant t$, есть вершины, которые были смежными вершинами $v_{1}^{\prime}$ или $v_{2}^{\prime}$. Эти компоненты соединяются каким-либо образом в одну с помощью $s-1$ ребер (хорд) так, чтобы разводка по-прежнему оставалась планарной (рис. 2 c). Если в графе $G_{2}$ появилась изолированная вершина, то она удаляется, а из трассировки удаляется покрывающая ее разводка.

(b) Ребро $e_{2}$ не образует петли. Ребро $e_{2}$ удаляется из графа $G_{2}$. Вершины $v_{1}$ и $v_{2}$ стягиваются в одну вершину $v^{\prime}$. Разводка $R^{\prime}$ вершины $v^{\prime}$ строится следующим образом: из разводок $R_{1}$ и $R_{2}$ удаляются вершины $v_{1}^{\prime}$ и $v_{2}^{\prime}$ и инцидентные им ребра. Полученные разводки $R_{1}^{\prime}$ и $R_{2}^{\prime}$ объединяются в одну разводку $R^{\prime}$ (для определенности пусть вершины разводки $R^{\prime}$ были помечены числами $1, \ldots, m_{1}$, а вершины разводки $R_{2}$ числами $1^{\prime}, \ldots, m_{2}^{\prime}$, вершины $v_{1}^{\prime}$ и $v_{2}^{\prime}$ имели номера 1 и $1^{\prime}$, тогда в разводке $R^{\prime}$ вершины будут расположены по окружности в порядке $2, \ldots, m_{1}, 2^{\prime}, \ldots, m_{2}^{\prime}$, в качестве ребер разводки $R^{\prime}$ берется объединение множеств ребер разводок $R_{1}^{\prime}$ и $R_{2}^{\prime}$ ). Соответствие между вершинами разводки $R^{\prime}$ и ребрами (концами петель) инцидентными вершине $v$ остается таким же, каким было соответствие между вершинами разводок $R_{1}^{\prime}$ и $R_{2}^{\prime}$ и ребрами (концами петель) инцидентными вершинам $v_{1}$ и $v_{2}$. Пусть разводка $R^{\prime}$ состоит из $t$ связных компонент, при этом в $s$ связных компонентах, $1 \leqslant s \leqslant t$, есть вершины, которые были смежными вершинам $v_{1}^{\prime}$ или $v_{2}^{\prime}$. Эти компоненты соединяются каким-либо образом в одну с помощью $s-1$ ребер так, чтобы разводка по-прежнему оставалась планарной (рис. $2 \mathrm{~d}$ ).

Рассмотрим теперь преобразование обобщенной схемы $S_{0}$, состоящее в том, что одной из переменных функции $f\left(x_{1}, \ldots, x_{n}\right)$, реализуемой схемой $S_{0}$, присваивается константа $\alpha \in\{0,1\}$. Пусть это переменная с номером $i$. При этом схема изменяется следующим образом. Изоляторы и разомкнутые контакты схемы нижнего слоя, покрытые $i$-й $R$-компонентой, удаляются, а покрывающие их ребра схемы верхнего слоя замыкаются в соответствии с описанным выше алгоритмом; проводники и замкнутые контакты схемы нижнего слоя, покрытые $i$-й $R$-компонентой, замыкаются, а покрывающие их ребра схемы верхнего слоя удаляются в соответствии с описанным выше алгоритмом. Рассмотрим следующие возможные случаи.

(a) Если нижний слой полученной схемы состоит из одной связной компоненты, то считаем преобразование законченным.

(b) Если нижний слой полученной схемы состоит более, чем из одной связной компоненты, и полюсы $A$ и $B$ находятся в различных компонентах, то заменяем схему $S_{0}$ на пустую схему.

(c) Если нижний слой полученной схемы состоит более, чем из одной связной компоненты, и полюсы $A$ и $B$ находятся в одной компоненте, то ребра компонент, не имеющих полюсов, удаляются, а покрывающие их ребра схемы верхнего слоя замыкаются. Затем удаляются все изолированные вершины.

Легко проверить, что полученная схема будет удовлетворять определению обобщенной схемы, реализующей функцию $f\left(x_{1}, \ldots, x_{i-1}, \alpha, x_{i+1}, \ldots, x_{n}\right)$. 


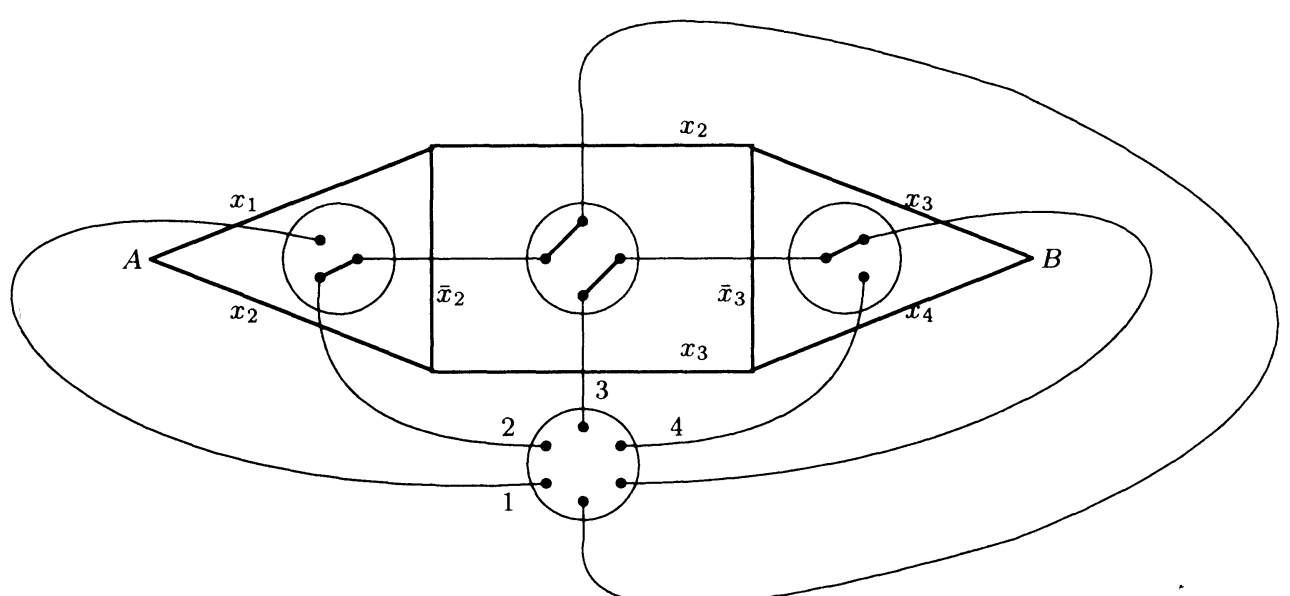

(a)

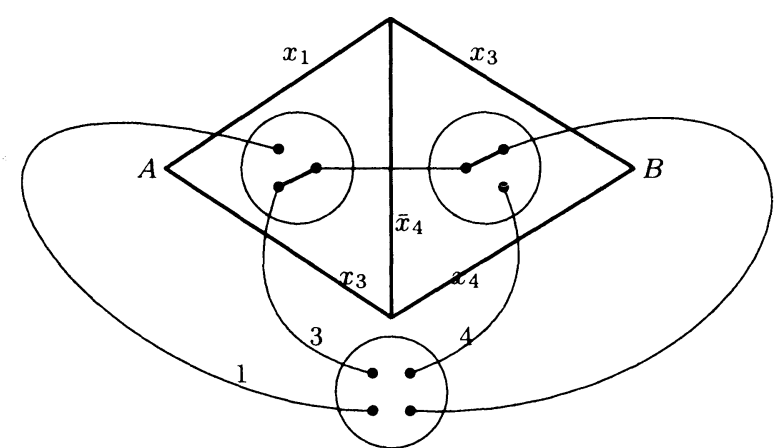

(b)

Рис. 3.

Пример такого преобразования приведен на рис. 3, где (a) - исходная схема, (b) - схема после подстановки $x_{2}=1$, вершины графа $G_{2}$ замещены соответствующими им разводками.

\section{4. Соотношение между функциями $L_{d}(f)$ и $L_{g}(f)$}

Целью данного параграфа является доказательство следующей леммы.

Лемма 1. Для любой булевой функции $f$ имеет место соотношение

$$
L_{d}(f) \geqslant \frac{1}{2} L_{g}(f) .
$$


Доказательство. Введем несколько моделей, промежуточных между схемами на целочисленной решетке и обобщенными схемами, и установим цепочку соотношений между сложностями реализации функций разными моделями.

4.1. Изменение меры сложности. Следующая модель будет отличаться от модели схем на целочисленной решетке тем, что за меру сложности мы будем принимать число ребер в схеме нижнего слоя. Сложность схемы, понимаемую таким образом, обозначим через $L_{p}(S)$. Очевидно, что

$$
L_{d}(f) \geqslant \frac{1}{2} L_{p}(f)
$$

4.2. Первое изменение схемы верхнего слоя. Каждую вершину схемы верхнего слоя покроем разводкой, которая строится следующим образом: вершины разводки, которым соответствуют проводники, соединяются в одну связную компоненту так, чтобы разводка по-прежнему оставалась планарной; остальные вершины разводки остаются изолированными. Затем заменим в схеме верхнего слоя все изоляторы на проводники и таким образом получим обобщенную схему верхнего слоя.

Сложностью схемы будем считать число ребер в схеме нижнего слоя и обозначать ее через $L_{T}(S)$. Нетрудно видеть, что

$$
L_{p}(f) \geqslant L_{T}(f)
$$

4.3. Второе изменение схемы верхнего слоя. По алгоритму, описанному в разделе 2, замкнем все ребра схемы верхнего слоя, которые не покрывают никаких ребер в схеме нижнего слоя. Сложностью схемы по-прежнему будем считать число ребер в схеме нижнего слоя и обозначать ее через $L_{c}(S)$. Как нетрудно видеть,

$$
L_{T}(f) \geqslant L_{c}(f) .
$$

Очевидно также, что только что введенные схемы являются частным случаем обобщенных схем. Поэтому

$$
L_{c}(f) \geqslant L_{g}(f)
$$

Из (1)-(4) вытекает утверждение леммы.

\section{5. Нижняя оценка}

Прежде, чем приступить непосредственно к получению нижней оценки, докажем несколько лемм.

Лемма 2. Число способов определить разводку вершины степени $m$ не превосходит $C_{1}^{m}$, где $C_{1}$ - некоторая константа. 
Доказательство. Разводка вершины представляет собой помеченный планарный граф без петель и кратных ребер специального вида. Обозначим через $r_{m}$ число различных разводок с $m$ вершинами, а через $q_{m}$ число урезанных разводок $\mathrm{c} m$ вершинами, то есть разводок, в которых не разрешается соединять ребром вершины с соседними номерами (номера 1 и $m$ также будем считать соседними). Очевидно, что

$$
r_{m} \leqslant 2^{m} q_{m}
$$

Нетрудно видеть, что $q_{1}=q_{2}=q_{3}=1$. Оценим $q_{m}, m \geqslant 4$, через $q_{3}, \ldots, q_{m-1}$. Число урезанных разводок, в которых вершина с номером 1 не является смежной никакой другой вершине, равняется $2 q_{m-1}$ (множитель 2 появляется из-за того, что вершины с номерами 2 и $m$ могут быть либо соединены ребром, либо нет). Число урезанных разводок, в которых вершина с номером 1 смежна вершине с номером $i, 3 \leqslant i \leqslant m-1$, равняется $q_{i} q_{m+2-i}$. Суммируя по всем возможным случаям, получаем, что

$$
q_{m} \leqslant 2 q_{m-1}+\sum_{i=3}^{m-1} q_{i} q_{m+2-i} \leqslant 3 \sum_{i=3}^{m-1} q_{i} q_{m+2-i} .
$$

Рассмотрим последовательность $p_{3}, p_{4}, p_{5}, \ldots$, определяемую следующим образом:

$$
p_{3}=1, \quad p_{m}=3 \sum_{i=3}^{m-1} p_{i} p_{m+2-i}, \quad m>3 .
$$

Очевидно, что при $m \geqslant 3$

$$
q_{m} \leqslant p_{m}
$$

Рассмотрим формальный числовой ряд

$$
P(t)=p_{3} t^{3}+p_{4} t^{4}+p_{5} t^{5}+\ldots+p_{m} t^{m}+\ldots
$$

Используя определение последовательности, нетрудно проверить, что

$$
3 P^{2}(t)=\left(P(t)-t^{3}\right) t^{2}
$$

откуда получаем, что

$$
P(t)=\frac{1}{6}\left(t^{2}-t^{2} \sqrt{1-12 t}\right)
$$

(побочный корень отбрасывается путем проверки условия $P^{(3)}(0)=6$, здесь $P^{(m)}(t)$ означает $m$-ю производную функции $P(t))$.

Ряд (7) представляет собой разложение функции $P(t)$ в ряд Маклорана, следовательно, $p_{m}=P^{(m)}(0) / m !$. Нетрудно проверить, что при $m \geqslant 4$

$$
P^{(m)}(0)=6^{m-3} m(m-1)(2 m-7) ! !
$$

где выражение $(2 m-1)$ !! означает $1 \cdot 3 \cdot 5 \ldots(2 m-1)$. Так как $(2 m-1)$ !! $<2^{m} m$ !, то $m(m-1)(2 m-7)$ !! $<2^{m} m$ !, и, следовательно, $p_{m}<12^{m}$. Учитывая (5) и $(6)$, а также то, что $q_{1}=q_{2}=q_{3}=1$, получаем оценку $r_{m} \leqslant 24^{m}$. Лемма доказана. 
Лемма 3. Число попарно неизоморфных связных планарных графов $c k$ ребрами не превосходит $C_{2}^{k}$, где $C_{2}-$ некоторая константа.

Доказательство см. в [5].

Две обобщенные схемы называются эквивалентными, если они реализуют одну и ту же функцию. Обозначим через $N(n, k)$ число попарно неэквивалентных схем, реализующих функции от $n$ переменных и имеющих сложность, не большую $k$.

Лемма 4. Справедливо неравенство

$$
N(n, k) \leqslant n ! C_{3}^{k}
$$

где $C_{3}-$ некоторая константа.

Доказательство. Оценим число попарно неэквивалентных схем, имеющих сложность $k$. Вообще говоря, один и тот же планарный граф можно уложить на плоскость по-разному так, что соответствующие двойственные графы будут неизоморфными. Поэтому две пары графов $\left(G_{1}, G_{2}\right)$ и $\left(G_{1}^{\prime}, G_{2}^{\prime}\right)$, где $G_{1}$ и $G_{1}^{\prime}$ - планарные графы, уложенные на плоскость, а $G_{2}$ и $G_{2}^{\prime}$ - двойственные к ним графы, будем считать эквивалентными, если изоморфны и графы $G_{1}$ и $G_{1}^{\prime}$, и графы $G_{2}$ и $G_{2}^{\prime}$. Из леммы 3 вытекает, что пару $\left(G_{1}, G_{2}\right)$ можно определить не более, чем $C_{2}^{2 k}$ способами (напомним, что граф $G_{2}$ имеет столько же ребер, сколько и граф $G_{1}$ ).

Далее, оценим число способов определения трассировки графа $G_{2}$. Пусть некоторая вершина $v$ имеет степень $m$. По лемме 2 ее разводку можно определить не более, чем $C_{1}^{m}$ способами. Сумма степеней всех вершин графа $G_{2}$ равна $2 k$, поэтому определить разводки всех вершин, а, следовательно, и трассировку можно не более, чем $C_{1}^{2 k}$ способами. Определение трассировки однозначно определяет разбиение множества ребер графа $G_{2}$ на максимальные $T$-компоненты. Из всех возможных разбиений отберем только те, в которых число $T$-компонент равно $n$. Занумеруем $T$-компоненты числами от 1 до $n$. Это можно сделать $n$ ! способами.

Граф $G_{1}$ имеет $k$ ребер и, значит, не более $2 k$ вершин. Следовательно, определить расположение обоих полюсов можно не более, чем $4 k^{2}$ способами. Каждому из $k$-ребер графа $G$ припишем один из символов множества $\{x, \bar{x}, 0,1\}$ (заметим, что индекс переменной определять не требуется, так как он определяется номером $T$ компоненты, ребро которой покрывает контакт). Это можно сделать $4^{k}$ способами.

Учитывая все сказанное, получаем, что число схем сложности $k$ не превосходит

$$
C_{1}^{2 k} C_{2}^{2 k} n ! 4 k^{2} 4^{k}=n ! 4 k^{2}\left(4 C_{1}^{2} C_{2}^{2}\right)^{k} .
$$

И, следовательно,

$$
N(n, k) \leqslant 1+\sum_{p=1}^{k} n ! 4 p^{2}\left(4 C_{1}^{2} C_{2}^{2}\right)^{p} \leqslant 1+n ! 4 k^{3}\left(4 C_{1}^{2} C_{2}^{2}\right)^{2} \leqslant n ! C_{3}^{k},
$$

где $C_{3}$ - некоторая константа. Лемма доказана.

Нижняя оценка сложности реализации обобщенными схемами одной функции будет получена методом Нечипорука [4]. Изложим этот метод в приложении к обобщенным схемам. 
Пусть множество $\tilde{x}$ переменных функции $f$ разбито на $p$ групп

$$
\tilde{x}_{1}, \tilde{x}_{2}, \ldots, \tilde{x}_{p}, \quad \bigcup_{i=1}^{p} \tilde{x}_{i}=\tilde{x}, \quad \tilde{x}_{i} \cap \tilde{x}_{j}=\varnothing, \quad i \neq j .
$$

Пусть далее $F_{i}$ обозначает множество различных функций, получающихся из функции $f$ при всевозможных подстановках констант на места всех переменных из $\tilde{x} \backslash \tilde{x}_{i}$.

Лемма 5. Справедливо неравенство

$$
L_{g}(f) \geqslant \sum_{i=1}^{p} h_{i}
$$

где $h_{i}$ есть наименъшее натуралъное число $h$, удовлетворяющее неравенству $\left|F_{i}\right| \leqslant$ $N\left(m_{i}, h\right)$, a $m_{i}$ - число переменных в множестве $\tilde{x}_{i}, i=1, \ldots, p$.

Доказательство. Пусть $l_{i}$ обозначает число ребер схемы нижнего слоя, покрытых ребрами компонент, соответствующих $i$-й группе переменных, в минимальной обобщенной схеме, реализующей функцию $f$. Каждой подстановке констант на места переменных из $\tilde{x} \backslash \tilde{x}_{i}$ соответствует серия преобразований, описанных в разделе 2 . Нижний слой полученной схемы имеет не более $l_{i}$ ребер. При различных подстановках констант должно получиться не менее $\left|F_{i}\right|$ различных схем. Таким образом, $l_{i}$ должно удовлетворять неравенству $\left|F_{i}\right| \leqslant N\left(m_{i}, l_{i}\right)$, поэтому $l_{i} \geqslant h_{i}$ и лемма доказана.

Пусть множество $\tilde{x}$ разбито на $p$ групп по $m$ переменных в каждой:

$$
\tilde{x}_{i}=\left\{x_{i 1}, \ldots, x_{i m}\right\}, \quad 1 \leqslant i \leqslant p,
$$

переменные $x_{i j}$ попарно различны,

$$
\bigcup_{i=1}^{p} \tilde{x}_{i}=\tilde{x}, \quad n=p m .
$$

Пусть далее $\tilde{\sigma}_{i j}$ - попарно различные булевы векторы длины $m$ и веса, большего 1. Обозначим через $K_{\tilde{\sigma}_{i j}}^{+}\left(\tilde{x}_{k}\right)$ конъюнкцию без отрицаний всех переменных из $\tilde{x}_{k}$, соответствующих единичным компонентам вектора $\tilde{\sigma}_{i j}$. Например, $K_{\tilde{\sigma}_{i j}}^{+}\left(\tilde{x}_{k}\right)=$ $x_{k 1} x_{k 4} x_{k 6}$ для $\tilde{\sigma}_{i j}=(100101)$.

Рассмотрим функцию

$$
f(\tilde{x})=\sum_{i j}\left(x_{i j} \sum_{k, k \neq i} K_{\tilde{\sigma}_{i j}}^{+}\left(\tilde{x}_{k}\right)\right),
$$

где суммирование проводится по модулю 2 . Положим $m=[\log n]+2$.

Лемма 6. $П р и ~ n \geqslant 6$

$$
\left|F_{i}\right|=2^{n-m}
$$

для всех $i, 1 \leqslant i \leqslant p$. 
Доказательство см. в [4].

Следствие 1. Справедливо неравенство

$$
L_{g}(f) \geqslant \frac{C_{4} n^{2}}{\log n},
$$

где $C_{4}-$ некоторая положительная постоянная.

Действительно, минимальное натуральное число $h$, удовлетворяющее неравенству

$$
2^{n-[\log n]-2} \leqslant([\log n]+2) ! C_{3}^{h},
$$

как нетрудно проверить, по порядку равно $n$. Остается учесть, что $p \sim n / \log n$, и воспользоваться леммой 5 .

Из следствия 1 и леммы 1 очевидным образом вытекает следуюшее утверждение.

Теорема. Справедливо неравенство

$$
L_{d}(f) \geqslant \frac{C n^{2}}{\log n}
$$

где $C$ - некоторая положительная постоянная.

Автор благодарит научного руководителя А. И. Рыбко за постановку задачи и помощь в работе, а также рецензента, сделавшего ряд полезных замечаний.

\section{Список литературы}

1. Таразевич Ю. Г. Реализация линейной функции плоскими контактными схемами и схемами на плоской целочисленной решетке. Дискретная математика (1992) 4, №1, 111-116.

2. Задорожнюк О. А., Рыбко А. И. Об одной модели плоских контактных схем. Дискретная математика (1995) 7, №4, 40-50.

3. Таразевич Ю. Г. Реализация функций контактными схемами на целочисленной решетке. В кн.: Тез. докл. конф. "Актуалъные проблемы социалъных, гуманитарных и естественных наук”, посвященной 70-летию БГУ. Вышэйшая школа, Минск, 1991, c.193-194.

4. Нечипорук Э. И. Об одной булевской функции. Докл. АН СССР (1966) 169, №4, 765-766.

5. Ветухновский Ф. Я. Об оценке числа плоских графов. Докл. АН $C C C P$ (1962) 142, №1, $50-53$. 